\title{
Rapid magnetic resonance imaging screening for abusive head trauma
}

\author{
Maura E. Ryan ${ }^{1}$ (D)
}

Received: 28 June 2019 /Revised: 28 June 2019 / Accepted: 16 July 2019 / Published online: 19 December 2019

(C) Springer-Verlag GmbH Germany, part of Springer Nature 2019

\begin{abstract}
Abusive head trauma (AHT) is the most common cause of fatal head injury in children younger than 2 years [1]. However, because clinical presentations of AHT are variable and accurate history is typically absent, assessment can be challenging and imaging is often crucial for diagnosis. Historically, CT has been the standard for initial evaluation, but the necessity of imaging must be weighed against concerns for radiation exposure in this vulnerable population, which could lead to underutilization of imaging in screening for AHT.
\end{abstract}

The article by Berger and colleagues [2] in this issue of Pediatric Radiology proposes using a non-sedated, limited rapid MR brain exam in lieu of CT to evaluate infants at risk for AHT. Employing a targeted protocol consisting of axial T2-W half-acquisition single-shot fast spin echo (ssFSE), diffusion-weighted imaging (DWI), gradient recalled echo (GRE), and coronal T1-weighted inversion recovery sequences, as well as a standard axial T2-W FSE sequence for comparison, the authors attempted to screen 158 infants for intracranial hemorrhage. The completion rate was $98 \%$ (3 cases were non-diagnostic from motion) and there was only a single instance of discrepancy between standard and singleshot T2 sequences. Furthermore, of 145 normal studies, only 8 infants underwent subsequent head $\mathrm{CT}$ or MR, avoiding radiation in a significant proportion of the screened infants.

Rapid MRI techniques centered on ultrafast T2-weighted sequences that can be performed without sedation initially gained popularity as a method of evaluating and following children with hydrocephalus to avoid radiation exposure from repeated head CTs $[3,4]$. The rapid protocol can typically be

Maura E. Ryan

mryan@luriechildrens.org

1 Department of Medical Imaging,

Ann \& Robert H. Lurie Children's Hospital of Chicago,

Northwestern University Feinberg School of Medicine,

225 East Chicago Ave,

Chicago, IL 60611, USA incorporated into a busy MRI workflow. The ease of scheduling and freedom from concerns of radiation or sedation effects have made this an increasingly popular technique for evaluating a variety of other indications, including trauma [5].

The article by Berger and colleagues [2] introduces an interesting potential application for rapid brain imaging in the setting of trauma - one that could decrease the rate of missed abusive head trauma by encouraging imaging use in at-risk but well-appearing children. Additionally, this technique would decrease the overall radiation exposure in at-risk infants. In Berger et al., $27 \%$ of the rapid brain studies were described as concerning for motion, although only $2 \%$ were deemed uninterpretable. Determining how much motion should be considered non-diagnostic might be heavily influenced by experience, but even a low threshold could still result in a substantial decrease in CTs performed.

However, in my opinion, the reliability and sensitivity of rapid MRI for traumatic head injury remains suboptimally tested. A few small studies have suggested that rapid MRI can be used to detect clinically significant intracranial injury that would require neurosurgical intervention, but the sensitivity for trace extra-axial hemorrhages of a few millimeters in width has been questioned. In one study of 54 children by Sheridan et al. [6], rapid brain MRI demonstrated 85\% sensitivity for all intracranial traumatic injuries, with small extraaxial hemorrhages missed in 4 children. At our institution, we previously evaluated 102 hemorrhages in children and demonstrated an overall sensitivity of $86 \%$ for subdural or epidural hemorrhages with rapid MRI [7]. It should be noted that neither study included T1 imaging, which was performed in the study by Berger and colleagues [2].

There are significant obstacles to interpreting these and other studies of rapid MRI used for trauma evaluation. As with many pediatric studies, the sample sizes are relatively small. The study by Berger et al. [2] is similarly limited by a small number of positive cases, with only 13 patients positive for traumatic injury. Furthermore, meta-analysis is confounded by a wide variability in technique. There is no single, uniform 
accepted protocol for rapid brain MR. Studies are generally centered on multiplanar rapid T2 sequences and trauma evaluation typically includes a heme-sensitive sequence such as GRE or susceptibility-weighted imaging (SWI), as well as DWI. However, other sequences such as T1 or T2 fluid-attenuated inversion recovery (FLAIR) are increasingly included, particularly as faster versions become commercially available.

Another potential pitfall in replacing CT with rapid MRI screening concerns the decreased sensitivity of MR for skull fractures. Berger et al. [2] proposed that patients screened for suspected abuse would also undergo a skeletal survey and that the skull films could be used to assess for fractures. However, CT is more sensitive for subtle fractures, especially with modern multichannel scanners that can quickly and effortlessly provide 3-D reformations. Nakahara et al. [8] concluded that plain radiographs might fail to detect as much as $21 \%$ of skull fractures identifiable by $\mathrm{CT}$ with reformations.

One last note - rapid MR should not be considered a replacement for complete standard MRI performed in children with equivocal findings on initial screening or those in need of further evaluation for treatment or medicolegal documentation. In these instances, the full complement of standard MR sequences, the possible addition of venographic imaging for bridging vein rupture and in some cases spine imaging for additional evidence of injury might be warranted.

Rapid MRI is a powerful emerging tool for safer intracranial imaging evaluation in the pediatric population. The ease and safety of fast imaging could play a role in improving detection of AHT in well-appearing children who might otherwise not be screened by imaging because of radiation concerns. However, the variations in technique and the relatively small size of published studies limit substantive evidencebased assessment of the true sensitivity and limitations. In evaluation of abusive head trauma, where subtle findings might have great clinical consequence, the adoption of a rapid MR screening protocol is promising but should be considered with caution.

\section{Compliance with ethical standards}

Conflicts of interest None

\section{References}

1. Choudhary AK, Servaes S, Slovis TL et al (2016) Consensus statement on abusive head trauma in infants and young children. Pediatr Radiol 48:1048-1106

2. Berger RP, Furtado A, Flom L et al (2019) Implementation of a brain injury screen MRI for infants at risk for abusive head trauma. Pediatr Radiol 46(4):519-526

3. Iskandar BJ, Sansone JM, Medow J, Rowley HJ (2004) The use of quick-brain magnetic resonance imaging in the evaluation of shunt treated hydrocephalus. J Neurosurg 101:147-151

4. Niederhauser BD, McDonald RJ, Keating GF et al (2013) Retrospective review of rapid pediatric brain MR imaging at an academic institution including practice trends and factors affecting scan times. AJNR Am J Neuroradiol 2013:1836-1840

5. Missios S, Quebada PB, Forero JA (2008) Quick-brain magnetic resonance imaging for nonhydrocephalus indications. J Neurosurg Pediatr 103:438-444

6. Sheridan DC, Newgard CD, Selden NR et al (2017) QuickBrain MRI for the detection of acute pediatric traumatic brain injury. J Neurosurg Pediatr 19:259-264

7. Ryan ME, Jaju A, Ciolino JD, Alden T (2016) Rapid MRI evaluation of acute intracranial hemorrhage in pediatric head trauma. Neuroradiology 58:793-799

8. Nakahara K, Shimizu S, Utsuki S et al (2011) Linear fractures occult on skull radiographs: a pitfall at radiological screening for mild head injury. J Trauma 70:180-182

Publisher's note Springer Nature remains neutral with regard to jurisdictional claims in published maps and institutional affiliations. 